\title{
BALANCED TRUNCATION OF PERTURBATIVE REPRESENTATIONS OF NONLINEAR SYSTEMS
}

\author{
Marissa Condon and Georgi Grahovski \\ School of Electronic Engineering and member \\ of RINCE, Dublin City University, Dublin 9, \\ IRELAND \\ marissa.condon@dcu.ie
}

KEY WORDS: controllability and observability gramians, model reduction, balanced truncation

\begin{abstract}
The paper presents a novel approach for a balanced truncation style of model reduction of a perturbative representation of a nonlinear system. Empirical controllability and observability gramians for nonlinear systems are employed to define a projection matrix. However, the projection matrix is applied to the perturbative representation of the system rather than directly to the exact nonlinear system. This is to achieve the required increase in efficiency desired of a reduced-order model. Application of the new method is illustrated through a sample test-system. The technique will be compared to the standard approach for reducing a perturbative representation of a nonlinear system.
\end{abstract}

\section{INTRODUCTION}

With the ever-growing complexity of dynamic models for systems in all branches of engineering, simulation times and use of computational resources have concomitantly increased to impractical levels. Consequently, the development of effective model reduction techniques is of paramount importance. In particular, for control system design for nonlinear systems eg Lall 2002, numerous iterative or repetitive simulations are required to meet target specifications. Hence, system representations in these studies must meet a required level of accuracy but with a low computation cost and time.

Model reduction for linear systems has received much attention for the past number of decades and several techniques are well-known and widely used (Antoulas 2001, 2003). Balanced Truncation, as developed and pioneered by Moore in 1981(Moore 1981), is one such method. In linear system theory (e.g. see (Antoulas 2001, 2003) and the references therein), the input-output interaction of a system is characterized by the so-called gramian matrices or gramians. These matrices can be transformed to be equal or balanced (Antoulas 2001, Moore 1981). For general nonlinear systems, the more general concept of controllability and observability (or energy) functions (Scherpen 1993, 1994) are employed for balancing. However, the calculation of the energy functions is computationally

\author{
Rossen Ivanov \\ Department of Mathematics, Trinity College \\ Dublin, IRELAND
}

expensive and the result is rarely an explicit solution (Scherpen 1993, Gray 1998). For these reasons, it is very difficult to apply this method to large-scale problems. To counteract this, empirical gramians were suggested in several recent research papers, (Lall 2002) followed by Hahn 2002 and Condon 2004. In (Condon 2004), some shortcomings of the approaches in (Lall 2002) and (Hahn 2002) were highlighted and an improved approach for the computation of the empirical gramians was suggested and confirmed with numerical tests.

There is one significant issue with the model reduction approach using empirical gramians as described in (Lall 2002, Hahn 2002 or Condon 2004). As stated in (Phillips 2003) and (Rewienski 2003) and as will be described in Section 4, it is dubious as to whether direct use of the projection matrix with the exact nonlinear function actually results in a significantly more efficient model for a nonlinear system. Therefore in this contribution, although the projection matrix is derived from empirical gramians obtained from the exact system representation, it is applied to a perturbative representation of the nonlinear system. This approach results in an efficient reducedorder model. Numerical results will then confirm that the approach is superior to the existing Krylov methods for reduction of perturbative representations.

\section{BALANCED TRUNCATION}

To review balanced truncation, consider the following standard linear system representation:

$$
\begin{gathered}
\dot{x}(t)=A x(t)+B u(t) \quad x \in \mathfrak{R}^{n}, u \in \mathfrak{R}, A \in \mathfrak{R}^{n \times n}, B \in \mathfrak{R}^{n} \\
y(t)=C x(t) \quad y \in \mathfrak{R}, C \in \mathfrak{R}^{n}
\end{gathered}
$$

$A$ is a constant matrix and $B$ and $C$ are constant vectors ( $C$ is a vector-row and $B$ is a vector-column). $u(t)$ is the input to the system and $y(\mathrm{t})$ is the output of the system. The input-output behaviour of the linear system is characterised by two matrices - the controllability and observability gramians. The matrices are defined as follows:

$$
\begin{aligned}
& P=\int_{0}^{\infty} e^{A t} B B^{*} e^{A^{*} t} d t \\
& Q=\int_{0}^{\infty} e^{A^{*} t} C^{*} C e^{A t} d t
\end{aligned}
$$


(* denotes transpose). $P$ and $Q$ are the solutions of the Lyapunov Equations (eqns. 4 and 5) and efficient techniques exist for their solution (Saad 1990).

$$
\begin{aligned}
& A P+P A^{*}=-B B^{*} \\
& A^{*} Q+Q A=-C^{*} C
\end{aligned}
$$

The subspace spanned by the eigenvectors corresponding to non-zero eigenvalues of $P$ contains the states that are reachable or controllable by the input to the system. The subspace spanned by the eigenvectors corresponding to non-zero eigenvalues of $Q$ contains the observable states at the output. The eigenvalues of the product $P Q$ are known as the Hankel Singular Values. By carrying out a transformation as described in e.g. (Lall 2002), it is possible to diagonalise the gramians and make them both equal. This particular arrangement is known as a balanced realisation. When a linear system is balanced, each Hankel Singular value represents a different state through which the input may be transferred to the output. Consequently, the states corresponding to the largest Hankel Singular values, being the most important, may be retained and those of relatively minor importance may be neglected.

Let $T$ be the matrix that transforms both $P$ and $Q$ into diagonal form $S$ as follows: $T P T^{t}=S, \quad T^{-1 t} Q T^{-1}=S \quad\left(T P Q T^{-1}=S^{2}\right)$. The states of the system are then ordered according to decreasing values of the diagonal entries in $S$. Once balanced, a Galerkin projection, $\Pi=\left[\begin{array}{ll}I & 0\end{array}\right]$ where $\Pi$ is $k \times n$ projection matrix and $I$ is $k \times k$ unit matrix, is then employed to project the transformed system onto the states corresponding to the $k$ largest singular values (i.e. the $k$ largest values of the diagonal matrix $S$ where $k$ is the desired dimension of the reduced-order model). The reduced model is thus of the form:

$$
\begin{aligned}
& \dot{z}(t)=\Pi T A T^{-1} \Pi^{*} z(t)+\Pi T B u(t) \\
& y(t)=C T^{-1} \Pi^{*} z(t)
\end{aligned}
$$

\section{EMPIRICAL GRAMIANS AND BALANCED TRUNCATION}

Now consider a non-linear system of the form:

$$
\begin{aligned}
& \dot{x}(t)=f(t, x(t))+B(t) u(t) \\
& y(t)=h(t, x(t))
\end{aligned}
$$

where $f: \mathfrak{R}^{n} \rightarrow \mathfrak{R}^{n}$ and $h: \mathfrak{R}^{n} \rightarrow \mathfrak{R}^{q}$ are non-linear functions, the function $u(t) \in \mathfrak{R}^{p}$ is regarded as an input signal to the system and the function $y(t) \in \mathfrak{R}^{q}$ is an output signal. For such a system, the more general concept of controllability and observability (or energy) functions (e.g. Scherpen 1993) would need to be employed for 'exact' balancing. However, the calculation of the energy functions is computationally expensive and the result is rarely an explicit solution (Scherpen 1993). For these reasons, it is very difficult to apply balanced truncation methods to large-scale nonlinear problems and to counteract this, empirical gramians were introduced (Lall 2002). In (Condon 2004), an improved approach for the computation of the empirical gramians was suggested and confirmed with numerical tests. In light of this, the 'improved' empirical gramians in (Condon 2004) are employed in this contribution and are defined as follows:

Let $\boldsymbol{M} \equiv\left\{c_{1}, \ldots, C_{s}\right\}$ be a set of $s$ positive constants, $\boldsymbol{T}^{\boldsymbol{n}} \equiv\left\{\boldsymbol{T}_{\mathbf{1}}, \ldots, \boldsymbol{T}_{\boldsymbol{r}}\right\}$ - be a set of $r$ orthogonal $n \times n$ matrices and $\boldsymbol{E}^{\boldsymbol{n}} \equiv\left\{\boldsymbol{e}_{1}, \ldots, \boldsymbol{e}_{\boldsymbol{n}}\right\}$ be the set of standard unit vectors in $\mathfrak{R}^{n}$. The set $\boldsymbol{E}^{\boldsymbol{n}}$ defines the standard directions and the set $\boldsymbol{T}^{\boldsymbol{n}}$ defines 'rotations' of these directions. The set $\boldsymbol{M}$ introduces different scales for each direction of the initial states.

\section{Definition 1}

Let $x^{\text {ilm }}(t)$ be the solution of eqn. 7 with $u \equiv 0$ :

$$
\dot{x}(t)=f(t, x(t))
$$

and with the initial condition:

$$
x^{\text {ilm }}(0)=c_{m} T_{l} e_{i}
$$

It is assumed that the initial condition (9) does not take the system outside the region of attraction of the equilibrium point $x=0$. The 'state-space average' of the 'nonlinear' fundamental solution may be defined as:

$$
\langle\Theta(t)\rangle=\frac{1}{r s} \sum_{m=1}^{s} \sum_{l=1}^{r} \sum_{i=1}^{n} \frac{1}{c_{m}} x^{i l m}(t) e_{i}^{*} T_{l}^{*}
$$

The nonlinear controllability gramian is then defined as:

$$
\widetilde{P}=\int_{0}^{\infty}\langle\Theta(-\tau)\rangle^{-1} B(-\tau) B^{T}(-\tau)\langle\Theta(-\tau)\rangle^{-1^{*}} d \tau
$$

where $\langle\Theta(t)\rangle$ is as described in (10).

Definition 2. For the system in (7) the nonlinear observability gramian is defined as:

$$
\widetilde{Q}=\int_{0}^{\infty} z^{*}(\tau) z(\tau) d \tau
$$

where $z(t) \in \mathfrak{R}^{n}$ is given by:

$$
z(t)=\frac{1}{r s} \sum_{i, l, m} \frac{1}{c_{m}} y^{i l m}(t) e_{i}^{*} T_{l}^{*}
$$

and $y^{i l m}(t)$ is the output which corresponds to an initial state $x^{i l m}(0)=c_{m} T_{l} e_{i}$ and a zero source term.

Now once the controllability and observability gramians are defined, the process of balancing may proceed as described in Section 2. The final reduced nonlinear model is given by:

$$
\begin{aligned}
& \dot{z}(t)=\Pi T f\left(t, T^{-1} \Pi^{*} z(t)\right)+\Pi T B(t) u(t) \\
& y(t)=h\left(t, T^{-1} \Pi^{*} z(t)\right)
\end{aligned}
$$

\section{PERTURBATIVE FORM OF THE NONLINEAR FUNCTION}

The reduction process in Section 3 and as used in (Lall 2002, Hahn 2002 and Condon 2004) involves application of the projection matrix directly to the exact nonlinear representation in eqn. 7 . However, since $f$ is a 
nonlinear function, the projection matrix may not in general be passed through the parentheses (Phillips 2003). Thus the evaluation of $\dot{z}(t)=\Pi T f\left(t, T^{-1} \Pi^{*} z(t)\right)$ necessitates initially setting $x(t)=T^{-1} \Pi^{*} z(t)$. The nonlinear function is then evaluated and finally the reduced space is formed using $\Pi T$ as $\Pi T f\left(t, T^{-1} \Pi^{*} z(t)\right)$. However, herein lies the problem. Evaluation of the function $f$ normally consumes up to $50 \%$ of the computing time in a nonlinear circuit simulation. Hence, although the state space size may be reduced significantly by setting $x(t)=T^{-1} \Pi^{*} z(t)$, the expected gain in computational efficiency will not be achieved.

As a consequence of this, approaches involving polynomial or perturbative forms and bilinear forms eg. (Phillips 2003 and Condon 2004) are employed. Several approaches for reduction via Krylov subspaces have been given eg. (Phillips 2003). However, in this contribution, it is proposed to (i) form a projection matrix from the empirical gramians derived from a full nonlinear system representation and then (ii) to employ the projection matrix with the perturbative representation of the system which is formed as follows:

Let $f(x)$ be expanded in a generalised Taylor's series about $x=0$ (the equilibrium point)

$$
f(x)=A_{1} x^{(1)}+A_{2} x^{(2)}+A_{3} x^{(3)}+\ldots
$$

where $x^{(1)}=x, \quad x^{(2)}=x \otimes x, \quad x^{(3)}=x \otimes x \otimes x$, etc. and $\otimes$ denotes the Kronecker product. Since $x=0$ is a stable equilibrium point, $A_{1}$ is a stable matrix. It is also assumed that each term in the Taylor's expansion is small compared to the previous one.

Now consider the case where a variational parameter $\quad \alpha \quad$ is introduced, i.e. $\dot{x}(t)=f(x(t))+B \alpha u(t)$ and let the response of the system $x(t)$ be perturbatively expanded in a power series in $\alpha$ (Phillips 2003):

$$
x(t)=\alpha x_{1}(t)+\alpha^{2} x_{2}(t)+\alpha^{3} x_{3}(t)+\ldots .
$$

On comparing terms in the variational parameter $\alpha$, the following set of $n$-dimensional differential equations can be derived:

$$
\begin{aligned}
& \dot{x}_{1}=A_{1} x_{1}+B u \\
& \dot{x}_{2}=A_{1} x_{2}+A_{2}\left(x_{1} \otimes x_{1}\right) \\
& \dot{x}_{3}=A_{1} x_{3}+A_{2}\left(x_{1} \otimes x_{2}+x_{2} \otimes x_{1}\right)+A_{3}\left(x_{1} \otimes x_{1} \otimes x_{1}\right)
\end{aligned}
$$

Each $n$-dimensional equation describes the time evolution of an $x_{i}$ where $x_{i}$ represents the $i^{\text {th }}$ order perturbative term in the expansion (16). Defining a vector $\underline{x}(t)$ :

$$
\underline{x}(t)=\left[\begin{array}{c}
x_{1} \\
x_{2} \\
\vdots
\end{array}\right]
$$

the system in (16) acquires the following form:

$$
\begin{aligned}
& \underline{\dot{x}}=\underline{A} \underline{x}+\underline{B} \underline{u} \\
& y=\underline{C} \underline{x}
\end{aligned}
$$

This form is termed the perturbative representation where

$$
\begin{aligned}
\underline{A} & =\left[\begin{array}{lllll}
A_{1} & & & \\
& A_{1} & & \\
& & A_{1} & \\
& & & \ddots
\end{array}\right] \underline{B}=\left[\begin{array}{ccccc}
B & 0 & 0 & 0 & \ldots \\
0 & A_{2} & 0 & 0 & \\
0 & 0 & A_{2} & A_{3} & \ldots \\
\vdots & \vdots & \vdots & \vdots
\end{array}\right] \underline{u}=\left[\begin{array}{c}
u_{1} \\
u_{2} \\
\vdots \\
\end{array}\right] \\
C & =\left[\begin{array}{lllll}
C & C & C & \ldots
\end{array}\right]
\end{aligned}
$$

$u_{1}=u(t), \quad u_{2}=x_{1} \otimes x_{1}$

The source $u_{2}$ for the second equation in (16) depends only on the state vector $x_{1}$ determined from the first equation and so on. Note that since $A_{1}$ is a stable matrix, $\underline{A}$ is also automatically stable. The system may be reduced using Krylov methods as described in (Phillips 2003). Section 5 will confirm that the utilisation of the projection matrix derived from the novel gramian definitions provides an alternative reduction method and results in greater accuracy for a test case.

\section{TEST CASE}

The example taken is a standard example for illustrating the effectiveness of model reduction techniques for nonlinear systems (e.g. Phillips 2003 and Chen 2000 and in many others). It consists of a network of nonlinear resistors and capacitors. The series of nonlinear resistors ensures a strong global nonlinearity. The nonlinear resistors are described by:

$$
i(v)=\left(e^{(40 v)}-1\right)+v
$$

The capacitors have unit capacitance. The input is a current source entering at node 1 and the output is the voltage at node 1 .

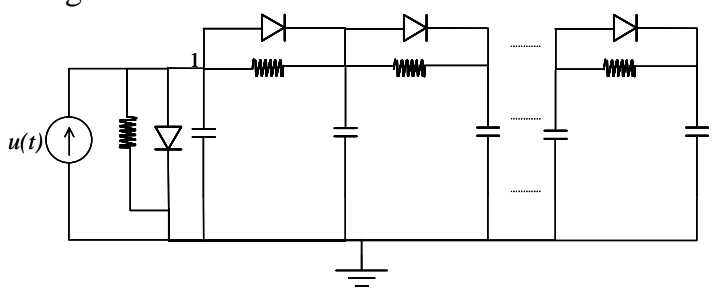

Fig. 1 Nonlinear $R C$ network

A ladder with 30 nodes is taken. The model is to be reduced to order 3 . A projection matrix is derived from the empirical gramians as described in Sections 2 and 3. Initially, the projection matrix is applied to the exact nonlinear representation and then the projection matrix is applied to the perturbative representation. The time for simulation of the latter is $\sim 17 \%$ of the former. Hence greater efficiency is achieved by using the perturbative representation (if the perturbative representation is deemed accurate enough for the design work in hand). Having established that use of the perturbative form is significantly more efficient, we 
now compare the reduction method to a standard Krylov approach (Phillips 2003). The solid line in Fig. 2 shows the exact perturbative result for the ladder network with an exponential input of the form $e^{-t}$. The dashed line gives the result achieved by using the Krylov method described by (Phillips 2003). In this method, the projection matrix is formed from $V_{1}$ and $V_{2} . V_{1}$ is computed such that its columns are a basis for $K_{q}\left(\left(s_{0} I-A_{1}\right)^{-1},\left(s_{0} I-A_{1}\right)^{-1} B\right)$ and $V_{2}$ is then defined as the basis for $K_{q}\left(\left(s_{0} I-A_{1}\right)^{-1},\left(s_{0} I-A_{1}\right)^{-1} B_{2}\right)$ where $A_{1}$ and $B$ are as defined in eqns. 16-19 and $B_{2}=A_{2}\left(V_{1} \otimes V_{1}\right)$. The dotted line gives the result obtained using the empirical gramians defined in eqns. 11 and 12. As is evident from the figure, the result obtained with the empirical gramians is clearly more accurate.

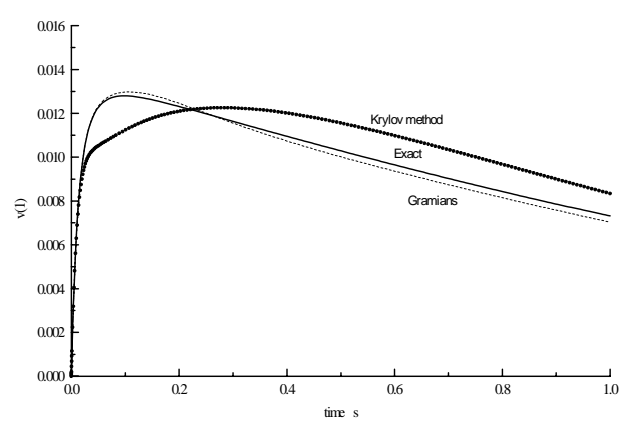

Fig. 2 Response at node 1 with an input of $e^{-t}$

\section{CONCLUSIONS}

The paper has recommended the empirical gramians introduced in (Condon 2004) for use in conjunction with a perturbative system representation to achieve an efficent and effective reduced model. By avoiding the direct use of the nonlinear function, the issue concerning the lack of the expected gain in computational speed with the use of a reduced model is avoided.

\section{ACKNOWLEDGEMENT}

The authors wish to acknowledge the financial support of Science Foundation Ireland.

\section{REFERENCES}

Antoulas AC, Sorensen DC, Gugercin S., 2001, 'A survey of model reduction methods for large-scale systems.' Contemporary Mathematics, AMS Publications, 2001, Vol. 280, pp. 193-219.

Antoulas A.C. 2003, Approximation of large-scale dynamical systems, SIAM Press- Philadelphia, 2003.

Chen Y, White J., 2000, 'A quadratic method for nonlinear model order reduction', International conference on modelling and simulation of Microsystems semiconductors, sensors and actuators, San Diego, 2000.

Condon, M. and Ivanov R., 2004, 'Empirical balanced truncation for nonlinear systems', Journal of Nonlinear Science, Oct. 2004
Condon, M. and R. Ivanov, R., 2005, ‘Balanced Model Reduction from a perturbative representation of weakly nonlinear systems', NOLTA 2005 Bruges, Belgium.

Gray WS, Scherpen JMA. 1998, 'Hankel Operators and Gramians for Nonlinear Systems', Proceedings of the 37th IEEE Conference on Decision and Control (CDC'98), Tampa, Fl, USA 1998, pp. 1416-1421.

Hahn J, Edgar TF, 2002, 'An Improved Method for Nonlinear Model Reduction Using Balancing of Empirical Gramians. Computers and Chemical Engineering 2002; Vol. 26(10), pp. 1379-1397.

Lall S, Marsden JE, Glavaški S., 2002, 'A subspace approach to balanced truncation for model reduction of nonlinear control systems', International Journal of Robust and Nonlinear Control 2002; Vol. 12, pp. 519-535.

Moore B., 1981, 'Principal Component analysis in linear systems: Controllability, Observability and model reduction', IEEE Trans. on Automatic Control 1981; AC-26(1).

Phillips JR., 2003, 'Projection-based approaches for model reduction of weakly nonlinear, time-varying systems', IEEE Transactions on computer-aided design of integrated circuits and systems 2003, Vol. 22, No. 2.

Rewienski M and White J., 2003, 'A trajectory piecewiselinear approach to model order reduction and fast simulation of nonlinear circuits and micromachined devices, IEEE Transactions on Computer-aided Design of Integrated Circuits and Systems 2003, Vol. 22, No. 2, pp.

Saad, Y., 1990, 'Numerical solution of large Lyapunov equations' Signal Processing, Scattering and Operator Theory, and Numerical Methods, Proc. MTNS-89, 3:503-511, Birkhauser,1990

Scherpen JMA.,1993, 'Balancing of nonlinear systems', Systems \& Control Letters 1993; Vol. 21, pp.143-153.

Scherpen JMA. 1994, 'Balancing of nonlinear systems', Dissertation, Systems and Control Group, University of Twente, Enschede, the Netherlands, 1994.

\section{AUTHOR BIOGRAPHIES}

MARISSA CONDON is a lecturer at DCU. Her research interests include the development of simulation techniques for nonlinear and high-frequency circuits and mathematical modelling of biological systems. Her webpage is /www.eeng.dcu.ie/ condonm.html. Her email is marissa.condon@dcu.ie

GEORGI GRAHOVSKI is a post-doctoral researcher at DCU. He has research interests in solitons, differential geometry and biophysical models in addition to nonlinear circuit simulation techniques.

ROSSEN IVANOV is a post-doctoral researcher at TCD. His research interests include nonlinear circuit simulation and differential equations solution techniques. Email: ivanovr@tcd.ie 